JGG 2022;70:144-151

doi: 10.36150/2499-6564-N471

\title{
Toll-like receptor 4 and the inflammation during aging
}

\author{
Aliakbar Yousefi-Ahmadipour ${ }^{1-3}$, Mohadeseh Sartipi ${ }^{1}$, \\ Hassan Khodadadii, ${ }^{4,5}$, Mehdi Shariati-Kohbanani ${ }^{1,6}$, \\ Mohammad Kazemi Arababadi ${ }^{1,2}$ \\ ${ }^{1}$ Immunology of Infectious Diseases Research Center, Research Institute of Basic Medical \\ Sciences, Rafsanjan University of Medical Sciences, Rafsanjan, Iran; ${ }^{2}$ Department of Laboratory \\ Sciences, Faculty of Paramedicine, Rafsanjan University of Medical Sciences, Rafsanjan, Iran; \\ ${ }^{3}$ Cancer and Stem Cell Research Laboratory, Faculty of Paramedicine, Rafsanjan University of \\ Medical Sciences, Rafsanjan, Iran; ${ }^{4}$ Geriatric Care Research Center, Rafsanjan University of Medical \\ Sciences, Rafsanjan, Iran; ${ }^{5}$ Department of Health and Sciences, Faculty of Health and Sciences, \\ Rafsanjan University of Medical Sciences, Rafsanjan, Iran; ${ }^{6}$ Department of Anatomy, Faculty of \\ Medicine, Rafsanjan University of Medical Sciences, Rafsanjan, Iran
}

Aging is significantly associated with inflammation, which contribute to the induction of several chronic inflammatory based diseases such as type 2 diabetes, age-related muscle wasting, and kidney dysfunctions. The detailed mechanisms involved in the induction of inflammation during aging are yet to be identified. However, it has been hypothesized that innate immunity sensors may be involved. Toll like receptors (TLRs) are a family of innate immunity sensors that play a significant role in the inducing inflammation in response to microbes or internal molecules. Therefore, TLRs can participate in the induction of inflammation. TLR4, as a member of the TLR family, is an important sensor that plays key roles in the detection of microbes and internal molecules in order to induce inflammation. Therefore, it may be hypothesized that TLR4 is a component of inflammation in the elderly population. The aim of this review is to explore the roles played by TLR4 in the induction/stimulation of inflammatory related complications during aging.

Key words: inflammation, Aging, TLR4

\section{Abbreviation}

BBB: blood-brain barrier

NF-kB: nuclear factor kappa-light-chain-enhancer of activated B cells

AP-1: activator protein 1

PBMC: peripheral blood mononuclear cell

ARMD10: age-related macular degeneration 10

MD2: myeloid differentiation 2

PAMP: pathogen associated molecular patterns

MHC: major histocompatibility complex

DAMP: damage associated molecular patterns

MYD88: myeloid differentiation primary response

TRIF: TIR-domain-containing adapter-inducing interferon- $\beta$

PRR: pathogen recognition receptor

TIR: toll/interleukin-1 receptor

LRRs: leucine-rich repeats

HSP60: heat shock protein 60

LPS: Iypopolysacharide 
HMGB-1: high-mobility group box-1

AMD: agerelated macular degradation

A $\beta$ : amyloid- $\beta$

SIGIRR: single immunoglobulin IL-1 (IL-1)-related receptor

CR: caloric restriction

POCD: postoperative cognitive dysfunction

DCs: dendritic cells

HF: fracture of the hip

WBV: whole body vibration

\section{INTRODUCTION}

Inflammation is much more common in the aged population than at any other stage of life ${ }^{1}$. It seems that either a reduction in the regulation of immune responses or an up-regulation/function of immune inducer molecules is the responsible mechanism that leads to the inflammation ${ }^{2}$. Accordingly, inflammation is one of the main factors associated with several agerelated diseases, including type 2 diabetes, age-related muscle wasting, atherosclerosis, kidney dysfunctions, Alzheimer's/Parkinson's diseases ${ }^{3,4}$. Researchers believe that non-microbial endogenous agents known as damage-associated molecular patterns (DAMPs) can induce inflammation during aging ${ }^{5,6}$. DAMPs have been reported to induce inflammation by interacting with their corresponding receptor known as the pathogen recognition receptors (PRRs) ${ }^{7}$. Toll-like receptors (TLRs) are important members of the PRRs expressed on the surface or intracellular vesicles of innate immune cells such as macrophages and dendritic cells ${ }^{7}$. TLR4 is an important PRR that recognizes a range of PAMPs and DAMPs and subsequently activates the immune system via up-regulation of major histocompatibility complexes (MHCs), pro-inflammatory cytokines, homing/ addressing molecules ${ }^{7}$. TLR4 induces the phenomenon via either myeloid differentiation primary response (MYD88) or TIR-domain-containing adapter-inducing interferon- $\beta$ (TRIF) dependent pathways ${ }^{8}$. TLR4 has been reported to be involved in the induction of CNS pathologies (such as dementia) by decreasing the process of remyelination ${ }^{9,10}$.

However, it has been hypothesized that TLR4 may be introduced as an important PRR involved in the induction of inflammation in the elderly population, because the antagonists of TLR4 can inhibit the interaction of ligands with TLR4 and block its intracellular signaling and decreased inflammation during aging ${ }^{11}$. Additionally, it has been proven that several aging complications like type 2 diabetes, cardiovascular diseases and kidney diseases have a potential link with TLR4 and its intracellular signaling molecules, which play an important role in the pathogenesis of the human diseases ${ }^{12-16}$. Thus, it seems that TLR4 can play key roles during aging inflammation. Therefore, current information on the state of TLR4 expression and its function during aging that can induce or stimulate inflammation is discussed in this review article.

\section{AGING}

Aging is the process of becoming older with progressive physiological changes ${ }^{17}$ that can lead to some complications ${ }^{18}$. This is a normal stage of human life and cannot be considered as a disease. Aged populations are increasing everywhere in the world and older adults are one of the most susceptible groups due to the incidence of mental and physical complications ${ }^{18}$.

\section{TLR4: STRUCTURE, GENE LOCATION, LIGANDS AND INTRACELLULAR PATHWAY}

Similar to other TLRs, the structure of TLR4 has three major domains starting at the $\mathrm{N}$-terminus. These include, leucine-rich repeats (LRRs), hydrophobic trans-membrane and cytoplasmic toll/interleukin-1 receptor (TIR) domains ${ }^{7}$. The TLR4 gene is located on chromosome 9q33.1 and is found in several mammal species ${ }^{19}$. TLR4 has other names in scientific literatures, including age-related macular degeneration10 (ARMD10) and CD284 ${ }^{7}$. As stated in our previous review article, that TLR4 recognizes a wide range of molecules as ligands, such as lypopolysacharide (LPS), high-mobility group box-1 (HMGB-1), heat shock protein 60 (HSP60), hyaluronan, allergenic nickel, free fatty acids and the adjuvant monophosphoryl lipid A (MPLA) ${ }^{7}$. Accordingly, it seems that both endogenous and exogenous ligands can interact with TLR4 and induce or stimulate immune responses and then subsequent inflammation ?

TLRs use two key adaptors, TRIF and MYD88, for transduction signals to immune cells following interactions with the corresponding ligands ${ }^{7}$. All TLRs use one of the adapter proteins, except TLR4, which uses both molecules ${ }^{7}$.

TLR4 interacts with its ligands at either on the cytoplasmic membrane or in the endosomes, resulting in activation of MYD88 or TRIF-dependent intracellular signaling pathways, respectively ${ }^{7}$. Accordingly, it has been hypothesized that the TLR4 signaling pathways can be activated via both pathogens and some endogenous molecules such as HMGB-1, HSPs and others, which are increased during aging ${ }^{20}$. Therefore, it has been proposed that TLR4 may be involved in the induction of the inflammation in the elderly population, as discussed in detail in the next section. 


\section{TLR4 AND INFLAMMATION DURING AGING}

Due to the important roles played by TLR4 in the induction of inflammation, it has been hypothesized that the molecule and its intracellular signaling may be involved in the induction of the inflammation in the elderly population ${ }^{21}$. Interestingly, recent information on both animal models and humans confirmed the hypothesis and revealed that TLR4 induces/stimulates inflammation in the population ${ }^{21}$. For instance, a study on human ARPE-19 cell line showed that subretinally-deposited amyloid- $\beta(A \beta)$ and its oligomeric forms $(O A \beta 1-42)$ play major roles in agerelated macular degradation (AMD), which often leads to irreversible blindness in the older adults ${ }^{11}$. Interestingly, the study reported that OA $\beta 1-42$ can activate TLR4 through both MYD88- and TRIF-dependent pathways ${ }^{11}$. Recent studies have reported that inhibition of the TLR4/MyD88 signaling pathway might protect aged rats against ischemia-reperfusion injury (IRI) in ischemic stroke ${ }^{22,23}$. In addition, as a TLR4 inhibitor, COBRA suppresses the expression of inflammatory molecules in response to OA $\beta 1-42$, leading to the improvement of $A M D{ }^{11}$. Another in vitro investigation by Caldeira et al. demonstrated that $A \beta$ upregulates TLR4 expression in young microglia, but not in old microglia, hence, implying that therapeutic approaches may differ from early to late-stage Alzheimer's disease ${ }^{24}$. Although the mentioned studies were performed under in vitro conditions, animal studies also confirmed the results ${ }^{25}$. Accordingly, aging and $A \beta$ oligomers have been shown to increase TLR4 expression and promote apoptosis in rat hippocampal neurons ${ }^{25}$. In addition, LPS-induced activation of the TLR4/NF- $\mathrm{kB}$ signaling pathway following microbiota-gut-brain axis dysfunction is related to age-related neuroinflammation and cognitive decline ${ }^{26}$. Therefore, as in vitro studies and animal models, it seems that TLR4 and its signaling pathways may be considered as a potential route to induce AMD ${ }^{24,25}$. Another animal study demonstrated that the production of LPS by gut microbiota will be increased with age and accelerates inflammation in the mice ${ }^{27}$. Based on the fact that LPS is one of the major ligands for TLR4, it may be concluded that gut microbiota LPS/TLR4 interaction is the mechanism leading to inflammation in the elderly population ${ }^{28}$. In parallel with the results it has been proven that the changes in the microbiota profile during aging are associated with inflammation and age-related diseases ${ }^{29}$. Recently, Neyrinck et al. reported the modulation of the gut microbiota, upregulation of TLR4 expression, and activation of the immune system in the ileum protect the old animals against liver inflammation ${ }^{30}$. Therefore, change in the microbiota profile may be considered as the reason for the alteration in roles played by TLR4 during aging. Moreover, based on the information present in this review article aging is associated with increased production of endogenous DAMPs ${ }^{27}$. In addition, aging is associated with augmented LPS-induced microglial activation; however, chronic exposure of the A $\beta$ to TLR4 can cause less responsive microglia, decrease $A \beta$ clearance, and thus accelerate $A D$ progression ${ }^{31}$. Another recent study on rat model reported aging predisposes brain tissue to be highly susceptible to LPS-induced brain microhemorrhages associated with increased blood-brain barrier (BBB) disruption and microglial/macrophage activation ${ }^{32}$. In this context, LPS-activation of endothelial cells via interaction with TLR4 leads to activation of the related signaling pathways and then cerebral cavernous malformations in a mouse model ${ }^{33}$.

Previous investigations revealed that the expression of single-immunoglobulin interleukin-1 (IL-1)-related receptor (SIGIRR) decreased in the older animals ${ }^{34}$. Moreover, SIGIRR is an inhibitory factor for the TLR4 signaling pathway ${ }^{34}$. A study by $\mathrm{Xu}$ et al. in rat model revealed that caloric restriction $(\mathrm{CR})$ attenuates inflammation in the elderly population via up-regulation of SIGIRR and then suppression of the TLR4 signaling pathway ${ }^{34}$. An increasing in the TLR4 expression in periodontitis, an age-related bone disease in non-human primates, has been proven by several studies ${ }^{35,36}$. A study by Zhong et al. on animal model demonstrated TLR4 deficiency ameliorates $\beta 2$-microglobulin induced age-related inflammation through suppression of the inflammatory factors $(\mathrm{IL}-1 \beta \text { and TNF- } \alpha)^{37}$. Postoperative cognitive dysfunction (POCD) is a complication of aging and recent data proposed that prophylactic lithium may have beneficial effects on the pathology of this disease ${ }^{38}$. Agrawal and colleagues demonstrated that increased production of an anti-inflammatory cytokine, IL-10, is impaired in response to lithium in old age ${ }^{39}$. The controversial data may be related to the age ranges and also the lithium doses. Additionally, in the POCD patients the inflammation was not considered as the effects of lithium. However, lithium chloride can induce young human dendritic cells (DCs) to produce IL-10, in a TLR4-dependent manner ${ }^{39}$. Hence, it seems that the roles played by TLR4 are different in the young when compared to old individuals. Senegenin, a traditional Chinese medicine, suppressed POCD in older rats via down-regulation of TLR4-induced MyD88 and TRIF-dependent signaling pathways ${ }^{40}$. Altogether, in vitro cell culture and animal models proved the roles of TLR4 in the pathogenesis of the age-related conditions, and thus the approaches and treatment strategies that are associated with decreased expression of TLR4 can improve geriatric disorders ${ }^{41-44}$. Consistent with the studies, human investigations also confirm the above hypothesis. For instance, a study by Wen et al. revealed that TLR4 is upregulated on the peripheral blood mononuclear cells (PBMCs) in a large population of old age $^{44}$. The upregulation of TLR4 seems to have potential 
effects on the neuronal functions in older adults. Downer et al. demonstrated that TLR4 expression increased in monocyte-derived macrophages from the IQ memory-discrepant (low-performing) group when compared to the IQ memory-consistent (high-performing) group ${ }^{45}$. Thus, it may be concluded that some of the age-related mental diseases, such as Alzheimer, may be affected by increased TLR4 expression and its corresponding inflammation. The results were confirmed by animal models. For example, Calvo-Rodríguez and colleagues reported that age-dependent TLR4 overexpression induces cell death in rat hippocampal neurons aged ${ }^{46}$.

Aging has been shown to be associated with oxidative stress, TLR4 expression, susceptibility to liver fibrosis, and high-fat diet (HFD)-induced liver inflammation in a mouse model ${ }^{47}$. In another animal model study, aging exacerbated HFD-induced glucose intolerance, decreased pancreatic $\beta$-cell function and survival, and induced tissue inflammation through TLR4 ${ }^{48}$. The study also demonstrated that TLR4 deficiency attenuates inflammatory macrophages and restores M2-like macrophage polarization in the liver and fat of aged mice ${ }^{48}$. The central role of TLR4 in adipose tissue inflammation during the aging process was confirmed by the study conducted by $\mathrm{He}$ and colleagues ${ }^{48}$. Additionally, there are several reports regarding the inflammatory roles of TLR4 in age-related disorders in the animal models ${ }^{2,49,50}$. Therefore, it seems that the animal models directed us to consider TLR4 as an important receptor involved in the pathogenesis of inflammation in the elderly population. Nevertheless, human models have also confirmed an important role for TLR4. Campbell and colleagues reported a TLR4-dependent increasing in platelet granzyme A, a serine protease that regulates monocyte-induced inflammatory gene synthesis and promotes inflammasome activation ${ }^{51}$.

Altogether, upregulation and TLR4 overexpression and its corresponding intracellular signaling pathways can be a risk factor for the induction or stimulation of inflammation in the elderly population and the subsequent induction of aging complications ${ }^{2,49,50}$. Figure 1 provides up-to-date information confirming the role of TLR4 and its associated intracellular signaling pathways in the pathogenesis of age-related complications. Further researches on the human models can improve knowledge of the molecular mechanisms of TLR4 in the pathogenesis of the elderly inflammation.

It has been proposed that resistance exercises may be beneficial for older adults and one of the major mechanisms may be via decreasing inflammation ${ }^{52}$. Rodriguez-Miguelez et al. showed that resistance exercise reduced human inflammation in the elderly population through inhibition of both TLR4 MyD88 and TRIF-dependent pathways ${ }^{53}$. Whole-body vibration (WBV) is also able to reduce the expression of TLR4, MyD88 and TRIF in older adults ${ }^{52}$. Based on the studies mentioned above, exercise and physical functions appear to help elderly individuals to lower inflammation, but there is a publication on animal model with contradicting results. Accordingly, Gomes et al. revealed that administration of nandrolone decanoate and performed resistance exercise training simultaneously were associated with higher occurrence of TLR4 activation in old animal ${ }^{54}$. Based on the fact that the studies done on humans and animals, and additionally the effects of exercise were not evaluated lonely, it may be hypothesized that the mechanisms of exercise in humans may be different from animals and administration of nandrolone decanoate may affect expression of TLR4. However, more human studies are needed to clarify our understanding of the physiology of aging.

Although most studies have confirmed the role of TLR4 in inflammation inducing in the elderly population, two animal studies and one human study have been associated with controversial results. A study on animal models showed decreased levels of TLR4 expression in old mice ${ }^{55}$. Aging also decreased TLR4 expression in mice gingival fibroblasts ${ }^{56}$. Fracture of the hip (HF) is a common complication in older adults and is a cause of morbidity and mortality ${ }^{57}$. After a thorough evaluation of the patients after a 6-month follow-up period, Bae$\mathrm{hl}$ and colleagues reported that HF did not alter TLR4 expression in the neutrophils of older adults ${ }^{57}$. Due to the results, it appears that the TLR4 roles during aging need more investigations.

\section{CONCLUSION REMARKS}

Due to the aforementioned investigations, it seems that age-related complications have a significant correlation with TLR4 expression and functions. Hence, it may be hypothesized that future therapeutic approaches could focus on the TLR4 antagonists using for treatment of inflammation in older human adults and its related complications. Additionally, due to the some controversial results between humans and animals, future studies under human and in vivo conditions need to be conducted to obtain more reliable scientific results.

\section{Ethical consideration}

This review article has been prepared as the ethical roles of The Ethical Committee of Iran University of Medical Sciences.

\section{Acknowledgement}

This project was supported by The Rafsanjan University of Medical Sciences. 


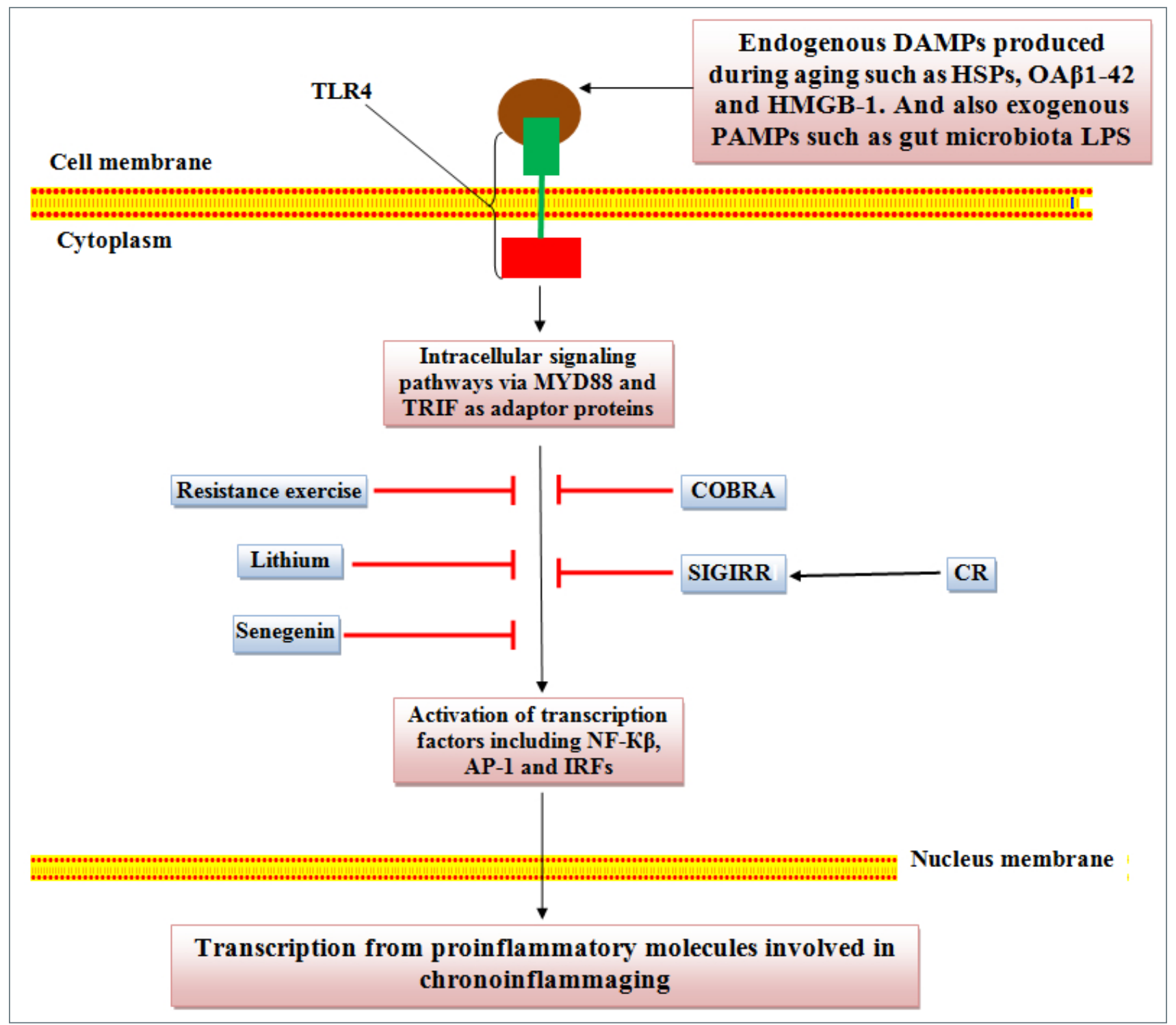

Figure 1. Roles played by TLR4 in induction of chronoinflammaging. Figure illustrates that interactions between PAMPs and DAMPs with TLR4 lead to activation of TLR4 intracellular signaling via both MYD88 and TRIF pathways which can result in chronoinflammaging. COBRA, SIGIRR, lithium, Senegenin and resistance exercise can modulate the signaling pathways and inhibit chronoinflammaging.

Conflict of interest

The Authors declare no conflict of interest.

\section{Funding}

This research did not receive any specific grant from funding agencies in the public, commercial, or not-forprofit sectors.

Informed consent

Obtained from the patient

Authors contribution

All the Authors contributed in the development of this manuscript.

\section{References}

1 Volt H, García JA, Doerrier C, et al. Same molecule but different expression: aging and sepsis trigger NLRP3 inflammasome activation, a target of melatonin. J Pineal Res 2016;60:193-205. https://doi.org/10.1111/jpi.12303

2 Chung HY, Kim DH, Lee EK, et al. Redefining chronic inflammation in aging and age-related diseases: proposal of the senoinflammation concept. Aging Dis 2019;10:367382. https://doi.org/10.14336/AD.2018.0324

3 Marogianni C, Sokratous M, Dardiotis E, et al. Neurodegeneration and Inflammation-an interesting interplay in Parkinson's disease. Int J Mol Sci 2020;21. https://doi. org/10.3390/ijms21228421 
4 Leng F, Edison P. Neuroinflammation and microglial activation in Alzheimer disease: where do we go from here? Nat Rev Neurol 2021;17:157-172. https://doi.org/10.1038/ s41582-020-00435-y

5 Pinti M, Cevenini E, Nasi M, et al. Circulating mitochondrial DNA increases with age and is a familiar trait: implications for " inflamm-aging". Europ J Immunol 2014;44:15521562. https://doi.org/10.1002/eji.201343921

6 Wang C, Klechikov AG, Gharibyan AL, et al. The role of proinflammatory S100A9 in Alzheimer's disease amyloid-neuroinflammatory cascade. Acta Neuropathol 12014;27:507522. https://doi.org/10.1007/s00401-013-1208-4

7 Zare-Bidaki M, Tsukiyama-Kohara K, Arababadi MK. Tolllike receptor 4 and hepatitis B infection: molecular mechanisms and pathogenesis. Viral Immunol 2014;27:321-326. https://doi.org/10.1089/vim.2014.0039

8 Strekalova T, Evans M, Costa-Nunes J, et al. TIr4 upregulation in the brain accompanies depression- and anxietylike behaviors induced by a high-cholesterol diet. Brain Behav Immun 2015;48:42-47. https://doi.org/10.1016/j. bbi.2015.02.015

9 Khan A, Park TJ, Ikram M, et al. Antioxidative and anti-inflammatory effects of Kojic acid in A-induced mouse model of Alzheimer's disease. Mol Neurobiol 2021;58:5127-5140. https://doi.org/10.1007/s12035-021-02460-4

10 Ved R, Sharouf F, Harari B, et al. Disulfide HMGB1 acts via TLR2/4 receptors to reduce the numbers of oligodendrocyte progenitor cells after traumatic injury in vitro. Sci Rep 2021;11:6181. https://doi.org/10.1038/ s41598-021-84932-0

11 Chen L, Bai Y, Zhao M, et al. TLR4 inhibitor attenuates amyloid-beta-induced angiogenic and inflammatory factors in ARPE-19 cells: Implications for age-related macular degeneration. Mol Med Rep 2016;13:3249-3256. https:// doi.org/10.3892/mmr.2016.4890

$12 \mathrm{Xu}$ Y, Jiang Z, Huang J, et al. The association between toll-like receptor 4 polymorphisms and diabetic retinopathy in Chinese patients with type 2 diabetes. Brit J Ophthalmol 2015;99:1301-1305. https://doi.org/10.1136/ bjophthalmol-2015-306677

13 Sepehri Z, Kiani Z, Nasiri AA, et al. Human Toll like receptor 4 gene expression of PBMCs in diabetes mellitus type 2 patients. Cell Mol Biol (Noisy-le-grand) 2015;61:92-95.

14 Candore G, Aquino A, Balistreri CR, et al. Inflammation, longevity, and cardiovascular diseases: role of polymorphisms of TLR4. Ann N Y Acad Sci 2006;1067:282-287. https://doi.org/10.1196/annals.1354.037

15 Zhao $\mathrm{H}$, Zheng $\mathrm{Q}, \mathrm{Hu} \mathrm{X}$, et al. Betulin attenuates kidney injury in septic rats through inhibiting TLR4/NF-кB signaling pathway. Life Sci 2016;144:185-193.

16 Zikou X, , Tellis CC, Rousouli K, et al. Differential membrane expression of toll-like receptors and intracellular cytokine induction in peripheral blood monocytes of patients with chronic kidney disease and diabetic nephropathy. Nephron Clinic Prac 2015;128:399-406.

17 McDonald RB. Basic concepts in the biology of aging. Biol Aging Garland Sci 2019;1-36.
18 Dues DJ, Andrews EK, Schaar CE, et al. Aging causes decreased resistance to multiple stresses and a failure to activate specific stress response pathways. Aging (Albany NY) 2016;8:777-795. https://doi.org/10.18632/ aging.100939

19 Xue X, Qiu Y, Jiang D, et al. The association analysis of TLR2 and TLR4 gene with tuberculosis in the Tibetan Chinese population. Oncotarget 2017;8:113082-113089. https://doi.org/10.18632/oncotarget.22996

20 Karuppagounder V, Giridharan W, Arumugam S, et al. Modulation of macrophage polarization and HMGB1TLR2/TLR4 cascade plays a crucial role for cardiac remodeling in senescence-accelerated prone mice. PloS One 2016;11:e0152922. https://doi.org/10.1371/journal. pone.0152922

21 Han D, Li F, Zhang H, et al. Mesencephalic astrocytederived neurotrophic factor restores blood-brain barrier integrity of aged mice after ischaemic stroke/reperfusion through anti-inflammation via TLR4/MyD88/NF-кB pathway. J Drug Target 2021;1-12. https://doi.org/10.1080/1 061186X.2021.2003803

22 Lv J, Yan W, Zhou J, et al. Per-and post-remote ischemic conditioning attenuates ischemic brain injury via inhibition of the TLR4/MyD88 signaling pathway in aged rats. Exp Brain Res 2021;239:2561-2567.

23 Sun Y, Geng J, Wang D. Cardioprotective effects of Ginsenoside compound-Mc1 and Dendrobium Nobile Lindl against myocardial infarction in an aged rat model: involvement of TLR4/NF-кB signaling pathway. Eur J Inflamm 2021;19:20587392211000577.

24 Caldeira C, Cunha C, Vaz AR, et al. Key aging-associated alterations in primary microglia response to beta-amyloid stimulation. Front Aging Neurosci 2017;9:277. https://doi. org/10.3389/fnagi.2017.00277

25 Calvo-Rodríguez M, de la Fuente C, García-Durillo M, et al. Aging and amyloid $\beta$ oligomers enhance TLR4 expression, LPS-induced $\mathrm{Ca}(2+)$ responses, and neuron cell death in cultured rat hippocampal neurons. J Neuroinflammation 2017;14:24. https://doi.org/10.1186/s12974-017-0802-0

26 Wu M-L, Yang X-Q, Xue L, et al. Age-related cognitive decline is associated with microbiota-gut-brain axis disorders and neuroinflammation in mice. Behav Brain Res 2021;402:113125.

27 Kim KA, Jeong JJ, Yoo SY, et al. Gut microbiota lipopolysaccharide accelerates inflamm-aging in mice. BMC Microbiol 2016;16:9. https://doi.org/10.1186/s12866-016-0625-7

28 Mouton PR, Kelley-Bell B, Tweedie D, et al. The effects of age and lipopolysaccharide (LPS)-mediated peripheral inflammation on numbers of central catecholaminergic neurons. Neurobiol Aging 2012;33:423 e427-436. https://doi. org/S0197-4580(10)00419-7

29 Santoro A, Zhao J, Wu L, et al. Microbiomes other than the gut: inflammaging and age-related diseases. Semin Immunopathol 2020;42:589-605. https://doi.org/10.1007/ s00281-020-00814-z 
30 Neyrinck AM, Taminiau B, Walgrave $H$, et al. Spirulina protects against hepatic inflammation in aging: an effect related to the modulation of the gut microbiota? Nutrients 2017;9. https://doi.org/10.3390/nu9060633

31 Go M, Kou J, Lim JE, et al. Microglial response to LPS increases in wild-type mice during aging but diminishes in an Alzheimer's mouse model: implication of TLR4 signaling in disease progression. Biochem Biophys Res Commun 2016;479:331-337. https://doi.org/10.1016/j. bbrc.2016.09.073

32 Sumbria RK, Grigoryan MM, Vasilevko V, et al. Aging exacerbates development of cerebral microbleeds in a mouse model. J Neuroinflammation 2018;15:69. https://doi. org/10.1186/s12974-018-1092-x

33 Tang AT, Choi JP, Kotzin JJ, et al. Endothelial TLR4 and the microbiome drive cerebral cavernous malformations. Nature 2017;545:305-310. https://doi.org/10.1038/ nature22075

$34 \mathrm{Xu}$ XM, Ning YC, Wang WJ, et al. Anti-Inflamm-aging effects of long-term caloric restriction via overexpression of SIGIRR to inhibit NF-kappaB signaling pathway. Cell Physiol Biochem 2015;37:1257-1270. https://doi. org/10.1159/000430248

35 Pandruvada SN, Gonzalez OA, Kirakodu S, et al. Bone biology-related gingival transcriptome in aging and periodontitis in non-human primates. J Clinic Periodont 2016;43:408-417.

36 Gonzalez OA, Novak MJ, Kirakodu S, et al. Differential gene expression profiles reflecting macrophage polarization in aging and periodontitis gingival tissues. Immunol Invest 2015;44:643-664. https://doi.org/10.3109/088201 39.2015.1070269

37 Zhong Q, Zou Y, Liu H, et al. Toll-like receptor 4 deficiency ameliorates $\beta 2$-microglobulin induced age-related cognition decline due to neuroinflammation in mice. Mol Brain 2020;13:20. https://doi.org/10.1186/s13041-020-0559-8

38 Zhao L, Wang F, Gui B, et al. Prophylactic lithium alleviates postoperative cognition impairment by phosphorylating

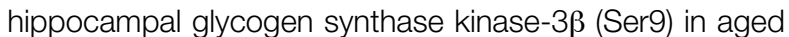
rats. Exp Gerontol 2011;46:1031-1036.

39 Agrawal S, Gollapudi S, Gupta S, et al. Dendritic cells from the elderly display an intrinsic defect in the production of $\mathrm{IL}-10$ in response to lithium chloride. Exp Gerontol 2013;48:1285-1292. https://doi.org/10.1016/j. exger.2013.08.006

40 Yu LY, Sun L, Chen SL. Protective effect of senegenin on splenectomy-induced postoperative cognitive dysfunction in elderly rats. Exp Ther Med 2014;7:821-826. https://doi. org/10.3892/etm.2014.1501

41 Song C, Zhang Y, Cheng L, et al. Tea polyphenols ameliorates memory decline in aging model rats by inhibiting brain TLR4/NF-кB inflammatory signaling pathway caused by intestinal flora dysbiosis. Exp Gerontol 2021;153:111476. https://doi.org/10.1016/j.exger.2021.111476
42 Zhang B, Lian W, Zhao J, et al. DL0410 alleviates memory impairment in D-galactose-induced aging rats by suppressing neuroinflammation via the TLR4/MyD88/NF-кB pathway. Oxid Med Cell Longev 2021:6521146. https:// doi.org/10.1155/2021/6521146

43 Liu H, Chu S, Wu Z. Loss of toll-like receptor 4 ameliorates cardiovascular dysfunction in aged mice. Immun Ageing 2021;18:42. https://doi.org/10.1186/ s12979-021-00251-y

44 Wen CJ, Chang CH, Chen CY, et al. Age-dependent messenger RNA expression of toll-like receptor 4 and intercellular adhesion molecule-1 in peripheral blood mononuclear cells. Eur J Clin Invest 2021;51:e13522. https://doi. org/10.1111/eci.13522

45 Downer EJ, Jones RS, McDonald CL, et al. Identifying early inflammatory changes in monocyte-derived macrophages from a population with IQ-discrepant episodic memory. PloS One 2013;8:e63194. https://doi.org/10.1371/journal.pone.0063194

46 Calvo-Rodríguez M, de la Fuente C, García-Durillo M, et al. Aging and amyloid $\beta$ oligomers enhance TLR4 expression, LPS-induced $\mathrm{Ca} \mathrm{2+}$ responses, and neuron cell death in cultured rat hippocampal neurons. J Neuroinflam 2017;14:1-13

$47 \mathrm{Kim} I \mathrm{H}, \mathrm{Xu}$ J, Liu X, et al. Aging increases the susceptibility of hepatic inflammation, liver fibrosis and aging in response to high-fat diet in mice. Age (Dordr) 2016;38:291-302. https://doi.org/10.1007/s11357-016-9938-6

48 Conaty P, Sherman LS, Naaldijk Y, et al. Methods of mesenchymal stem cell homing to the blood-brain barrier. In: Singh SR, Rameshwar P, eds. Somatic Stem Cells, vol. 1842. New York, NY: Springer, pp. 81-91.

49 Ao L, Zhai Y, Jin C, et al. Attenuated recovery of contractile function in aging hearts following global ischemia/ reperfusion: Role of extracellular HSP27 and TLR4 Mol Med 2017;23:863-872. https://doi.org/10.2119/ molmed.2016.00204

50 Pioli PD, Casero D, Montecino-Rodriguez E, Morrison SL, et al. Plasma cells are obligate effectors of enhanced myelopoiesis in aging bone marrow. Immunity 2019;51:351366 e356. https://doi.org/10.1016/j.immuni.2019.06.006

51 Campbell RA, Franks Z, Bhatnagar A, et al. Granzyme A in human platelets regulates the synthesis of proinflammatory cytokines by monocytes in aging. J Immunol 2018;200:295304. https://doi.org/10.4049/jimmunol.1700885

52 Rodriguez-Miguelez P, Fernandez-Gonzalo R, Collado PS, et al. Whole-body vibration improves the antiinflammatory status in elderly subjects through toll-like receptor 2 and 4 signaling pathways. Mechanisms Ageing Develop 2015;150:12-19. https://doi.org/10.1016/j. mad.2015.08.002

53 Rodriguez-Miguelez P, Fernandez-Gonzalo R, Almar M, et al. Role of Toll-like receptor 2 and 4 signaling pathways on the inflammatory response to resistance training in elderly subjects. Age (Dordr) 2014;36:9734. https://doi. org/10.1007/s11357-014-9734-0 
54 Gomes FC, Chuffa LG, Scarano WR, et al. Nandrolone decanoate and resistance exercise training favor the occurrence of lesions and activate the inflammatory response in the ventral prostate. Andrology 2016;4:473-480. https:// doi.org/10.1111/andr.12162

55 Sharma R, Kapila R, Haq MR, et al. Age-associated aberrations in mouse cellular and humoral immune responses. Aging Clin Exp Res 2014;26:353-362. https://doi. org/10.1007/s40520-013-0190-y
56 Domon H, Tabeta K, Nakajima T, et al. Age-related alterations in gene expression of gingival fibroblasts stimulated with Porphyromonas gingivalis. J Periodontal Res 2014;49:536-543. https://doi.org/10.1111/jre.12134

57 Baehl S, Garneau H, Le Page A, et al. Altered neutrophil functions in elderly patients during a 6-month follow-up period after a hip fracture. Exp Gerontol 2015;65:58-68. https://doi.org/10.1016/j.exger.2015.03.009 